\title{
Lipomatose epidural idiopática familiar: relato de três casos e revisão da literatura
}

\section{Familiar Idiopathic Spinal Epidural Lipomatosis: Report of Three Cases and Review of the Literature}

Cléciton Braga Tavares ${ }^{1}$ Emerson Brandão Sousa ${ }^{1}$ Igor Brenno Campbell Borges ${ }^{2}$

Alessandro F. Cardoso ${ }^{2}$ Rodrigo Carvalho Diniz ${ }^{3}$ Francisca das Chagas Sheyla Almeida Gomes Braga ${ }^{4}$

\author{
Address for correspondence Cléciton Braga Tavares, MD, \\ Universidade Estadual do Piauí, Faculdade de Ciências Médicas, Conjunto \\ lapep, Bloco C, ap. 32, Ilhotas, Teresina, PI, Brasil CEP: 64015-040 \\ (e-mail: bragatavares@yahoo.com.br). \\ Unidade de Neurocirurgia do Hospital de Base do Distrito Federal, DF, \\ Brasil; Faculdade de Ciências Médicas, Universidade Estadual do Piauí, \\ Teresina, Piauí, Brasil.
}

\section{Palavras-Chave \\ - lipomatosis \\ - espinal \\ - epidural \\ - idiopática \\ - paraparesia}

Lipomatose epidural é a doença causada pelo aumento da quantidade de gordura no espaço epidural da coluna vertebral, levando à compressão medular ou radicular. A medula toracolombar é a mais comumente envolvida. É geralmente encontrada em homens. As principais causas são a obesidade, altos níveis de corticoides exógenos ou endógenos e hipotireoidismo. A forma idiopática é rara, correspondendo a $17 \%$ dos casos. Manifesta-se como mielopatia compressiva ou das raízes da cauda equina. A ressonância magnética é o exame de escolha; sugere-se o diagnóstico quando a espessura da camada de gordura epidural ultrapassa $6 \mathrm{~mm}$. Nós adotamos neste caso a laminectomia com ressecção da gordura epidural, citada como uma das possíveis formas de tratamento. A maioria dos trabalhos demonstra melhora gradual, acentuada ou completa, do déficit neurológico nos primeiros 2 anos após a cirurgia.

Epidural lipomatosis is a disease caused by increased fat content in the epidural space of the spine, leading to root or spinal cord compression. The thoracolumbar spinal cord is the most commonly involved. It is usually found in men. The main causes are obesity, high levels of exogenous or endogenous steroids and hypothyroidism. The idiopathic form is rare, accounting for $17 \%$ of cases. It manifests as compressive myelopathy or radiculopathy. MRI is the test of choice, suggests the diagnosis when the thickness of the epidural fat exceeds $6 \mathrm{~mm}$. We adopt in this case laminectomy with fat resection, cited as one of the possible forms of treatment. Most studies show a gradual improvement, marked or complete neurological recovery in the first two years after surgery. received

May 1, 2015

accepted

June 12, 2015
DOI http://dx.doi.org/

$10.1055 / \mathrm{s}-0035-1559892$. ISSN 0103-5355.
Copyright $\odot 2015$ by Thieme Publicações License terms Ltda, Rio de Janeiro, Brazil
(®) $\Theta \circledast$ 


\section{Introdução}

Lipomatose epidural (LE) é a doença causada pelo aumento da quantidade de gordura no espaço epidural da coluna vertebral, levando à compressão medular ou das raízes da cauda equina. A medula torácica e lombar são as mais comumente envolvidas. $^{1-5}$

É geralmente encontrada em homens. As principais causas são a obesidade, altos níveis de corticoides exógenos ou endógenos e hipotireoidismo. A forma idiopática é rara, correspondendo a $17 \%$ dos casos. ${ }^{1,3,6}$ Apesar de descrita a relação entre a condição e o excesso de corticoides, até 2002 apenas dois casos de LE associados à síndrome de Cushing haviam sido publicados na literatura. ${ }^{7}$

O objetivo deste artigo é apresentar uma condição rara, mas importante, de déficit neurológico por compressão mielorradicular. Há apenas 16 casos publicados da forma idiopática, e este é o primeiro registro na literatura de LE idiopática familiar.

\section{Relato dos Casos}

\section{Caso 1}

Paciente R.M.B., 18 anos, masculino, longilíneo. Seis meses antes da admissão iniciou quadro de diminuição da força muscular nos membros inferiores, com dificuldade de deambular e piora progressiva. Chegou ao hospital em paraparesia e com retenção urinária intermitente. Referiu quadro semelhante em seu pai e em seu tio paterno. Negava uso de corticoides e não havia relato de hipotireoidismo ou doença de Cushing.

Ao exame, apresentava-se com hipertonia associada à paraparesia, força de grau III no membro inferior direito
(MID) e grau IV no membro inferior esquerdo, com hiperreflexia simétrica patelar e aquileu bilateralmente. Presença de clônus esgotável e Babinsk bilateralmente. Hipopalestesia simétrica em membros inferiores, mas com cinestesia inalterada. Marcha ceifante bilateral.

A ressonância da coluna vertebral evidenciou estreitamento do canal raquidiano no nível torácico superior e médio, com redução do líquido cefalorraquidiano. Maior nível de compressão medular em T5 (-Fig. 1).

Foi submetido a abordagem da coluna torácica por via posterior, com laminectomia de $\mathrm{T} 5$ a T9, e retirada de todo o tecido gorduroso visualizado na região posterior do canal, que comprimia de forma importante a medula espinhal. $\mathrm{O}$ exame histopatológico confirmou tratar-se de tecido adiposo (-Fig. 2).

Após 2 anos de segmento ambulatorial, o paciente apresentou resolução completa do déficit neurológico.

\section{Caso 2}

M.B.S., masculino, longilíneo, 45 anos de idade e pai do paciente anteriormente descrito no caso 1 . Apresentou-se com perda de força e dificuldade de movimentação do MID iniciada aos 18 anos de idade, com evolução progressiva desde então. Negava uso de corticoides e não havia relato de hipotireoidismo ou doença de Cushing.

Ao exame, apresentava-se com paresia de MID (força de grau IV) com hiperreflexia patelar e aquiliana ipsilaterais. Clônus esgotável à direita. Reflexo cutâneoplantar indiferente bilateral. Presença de marcha ceifante à direita. A ressonância evidenciava aumento da gordura epidural no nível torácico superior e médio (-Fig. 3). No entanto, o paciente recusou o tratamento cirúrgico.

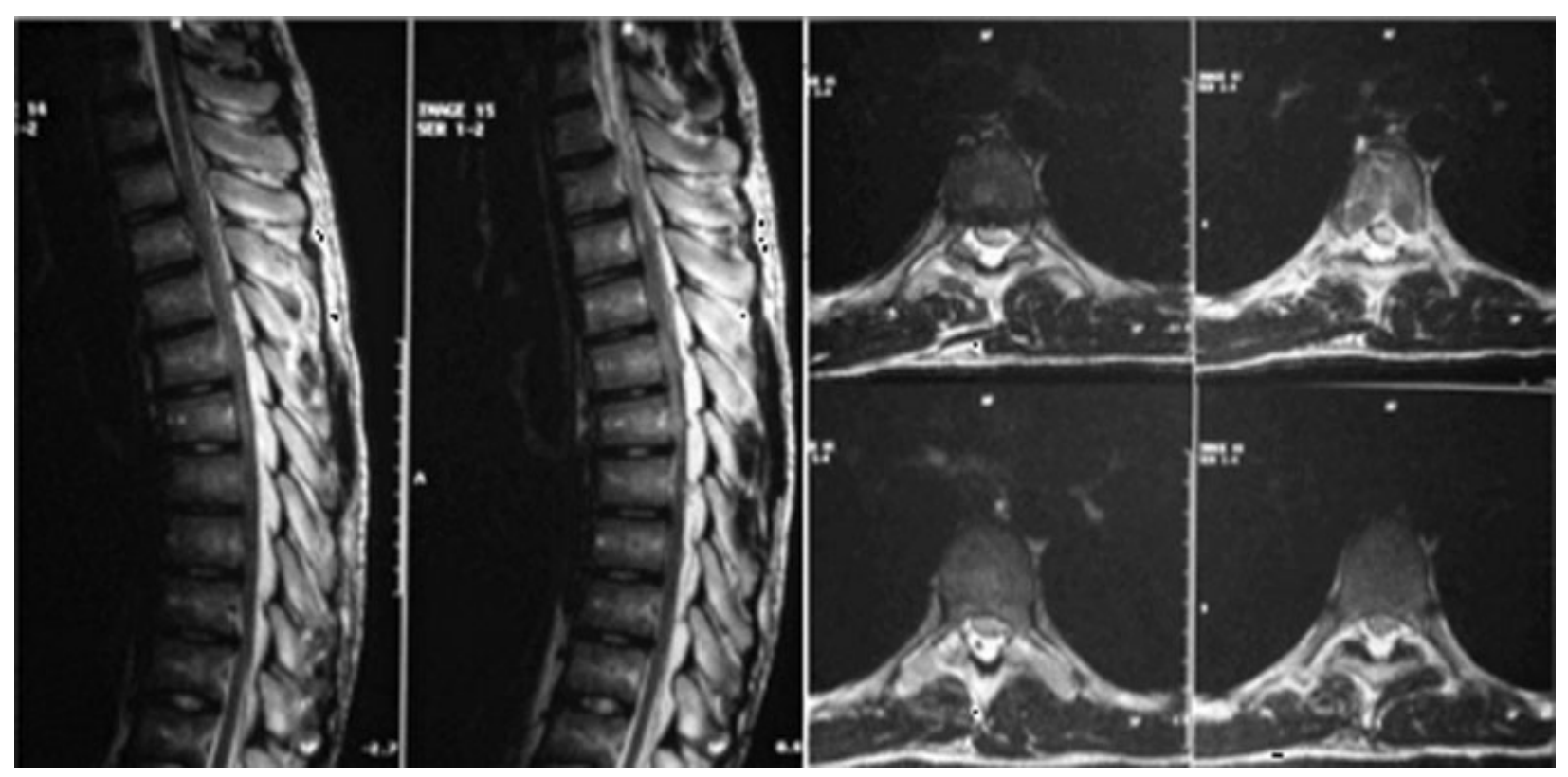

Fig. 1 Paciente R.M.B. Ressonância da coluna torácica (T2) evidenciando hiperintensidade intrarraquiana e extradural (lipomatose) com compressão medular. 


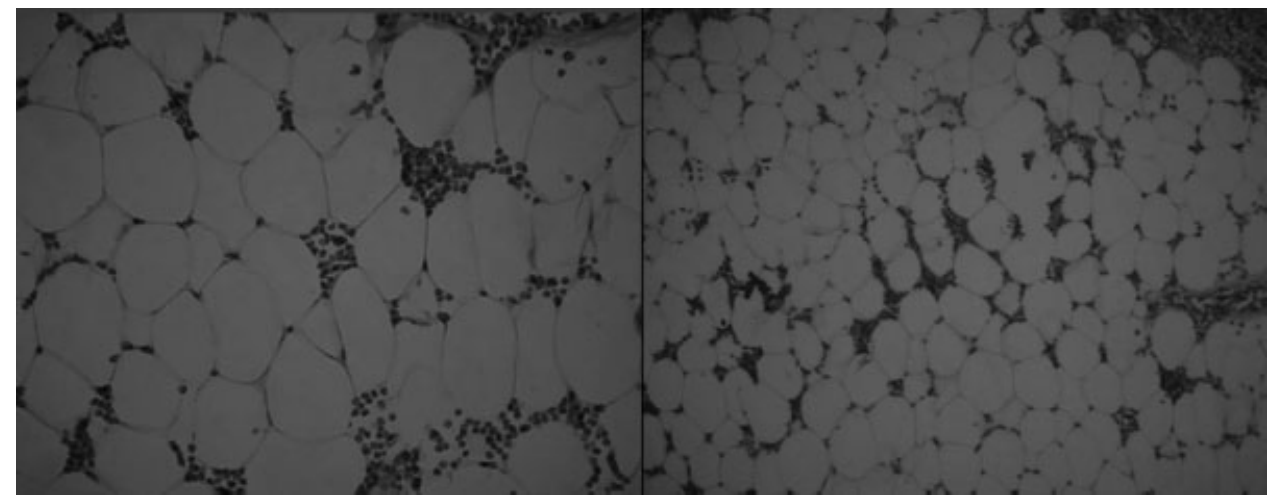

Fig. 2 Paciente R.M.B. Exame histopatológico, evidenciando grande quantidade de adipócitos.

\section{Caso 3}

E.C.B.B., masculino, longilíneo, 34 anos, tio paterno do paciente descrito no caso 1 . Apresentava fraqueza progressiva de membros inferiores há cerca de 12 anos. Negava uso de corticoides e não havia relato de hipotireoidismo ou doença de Cushing.

Ao exame, apresentava-se com paraparesia espástica (força de grau IV), hipoestesia tátil e dolorosa com nível sensitivo em T4, hipopalestesia e hipocinestesia crural, ataxia sensitiva e sinal de Roomberg presente. A ressonância evidenciava aumento da gordura epidural em toda a extensão da coluna torácica ( - Fig. 4).

Foi submetido a laminectomia de T4-T5-T6-T7 e ressecção de todo o tecido gorduroso visualizado na região posterior do canal que comprimia de forma importante a medula espinhal. O exame histopatológico confirmou tratar-se de tecido adiposo (-Fig. 5).

Após 2 anos de seguimento ambulatorial mantém paraparesia com força de grau IV e nível sensitivo em T4.

\section{Discussão}

A evolução crônica dos pacientes portadores de lipomatose epidural idiopática familiar é condizente com a literatura. Formas agudas de apresentação são raras. Manifesta-se principalmente como mielopatia compressiva, com paraparesia, nível sensitivo e retenção urinária; ou como radiculopatia, com dor, parestesias e claudicações. ${ }^{2,4}$ Há relatos de LE idiopática expressando-se como síndrome da cauda equina. ${ }^{6}$ Akhaddar et $\mathrm{al}^{8}$ descreveram um raro caso de LE idiopática que evoluiu com períodos de surto e remissão.

A lipomatose pode levar a déficit neurológico devido a compressão direta sobre a medula espinhal ou isquemia medular ocasionada por congestão venosa no interior do canal vertebral. ${ }^{9}$

Em todos os casos, podemos observar um excesso de tecido gorduroso no espaço epidural, caracterizado pela hiperintensidade de sinal em T2 na ressonância magnética (-Figs. 1, 3 e 4). A ressonância é o exame de escolha,

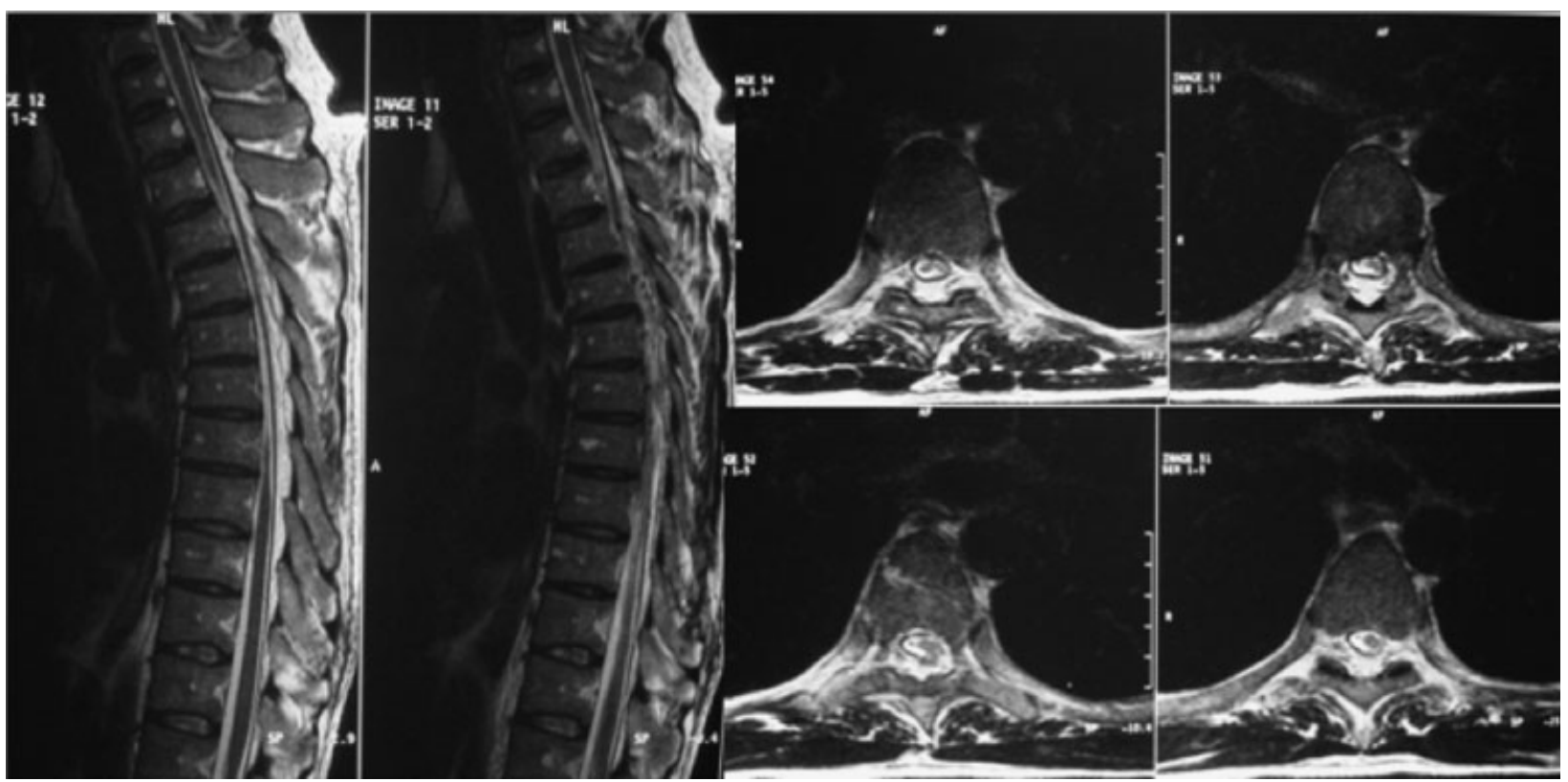

Fig. 3 Paciente M.B.S. Ressonância da coluna torácica (T2) evidenciando hiperintensidade intrarraquiana e extradural (lipomatose) com compressão medular. 


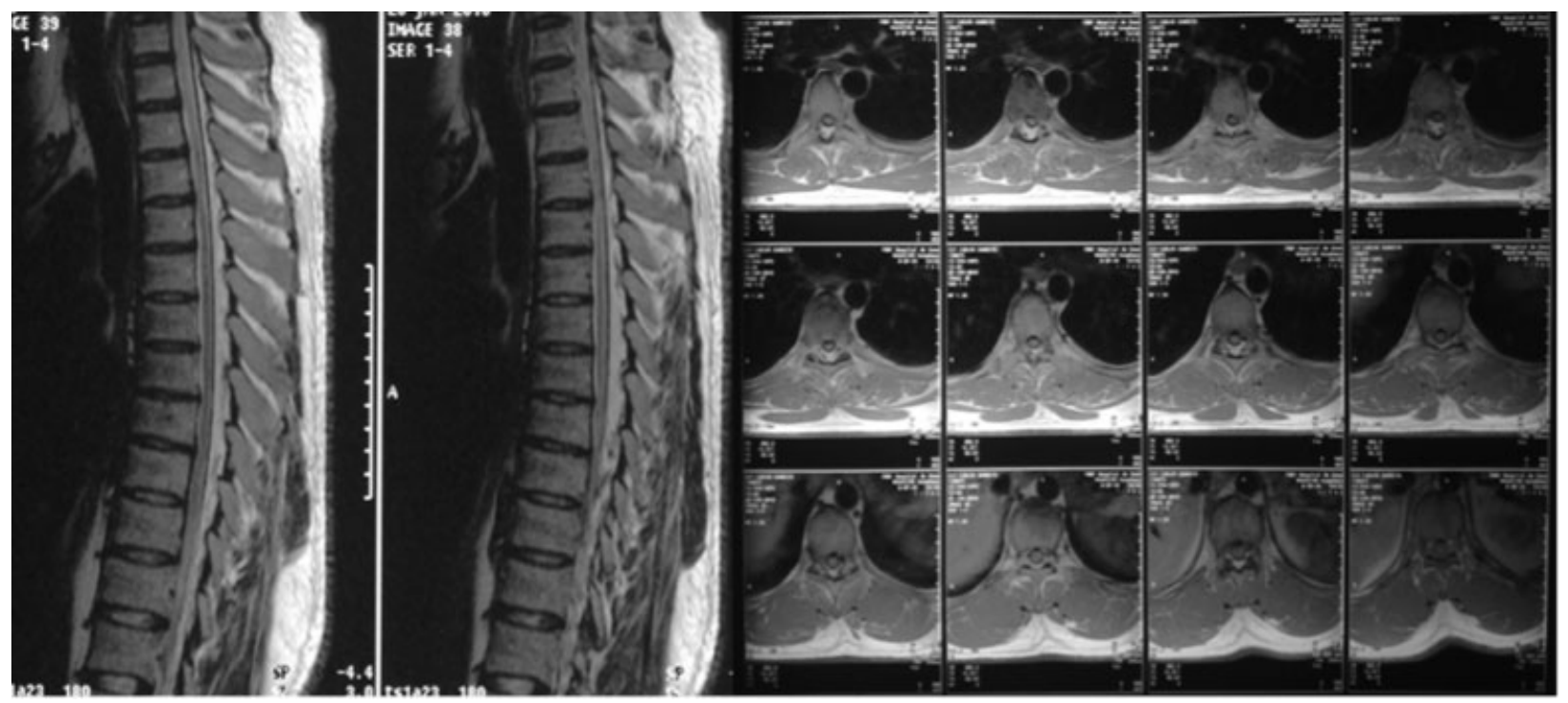

Fig. 4 Paciente E.C.B.B. Ressonância da coluna torácica (T2) evidenciando hiperintensidade intrarraquiana e extradural (lipomatose) com compressão medular.

sugerindo o diagnóstico quando a espessura da camada de gordura epidural ultrapassa $6 \mathrm{~mm} .{ }^{10-12}$ Tomografia e mielografia podem ser utilizadas para o diagnóstico, mas são menos eficazes. ${ }^{3} \mathrm{O}$ estudo do líquor pode evidenciar um aumento no número de proteínas, que significa apenas bloqueio de circulação liquórica. ${ }^{2}$

Nos três casos descritos não havia históricos de endocrinopatias (hipotireoidismo ou hipercortisolismo), sugerindo uma etiologia idiopática para o problema. LE idiopática foi primeiramente descrita por Badami e Hinck em 1982. ${ }^{13}$ Haddad et $\mathrm{al}^{14}$ foram os primeiros a propor a hipótese que a LE idiopática era secundária à obesidade que leva ao crescimento gradual do tecido gorduroso no espaço extradural raquidiano. No entanto, baseado nos conceitos atuais, 0 termo idiopático somente pode ser utilizado para lipomatoses em pacientes não obesos, que não utilizam corticoides exógenos e que não possuam endocrinopatias como hipotireoidismo ou hipercortisolismo endógeno. Apenas 16 casos em pacientes não obesos foram descritos até o momento na literatura. ${ }^{9}$

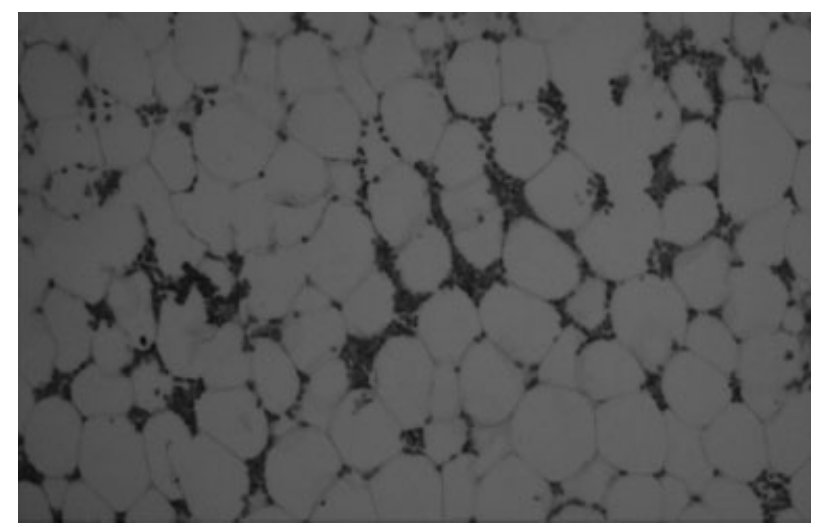

Fig. 5 Paciente E.C.B.B. Exame histopatológico, evidenciando grande quantidade de adipócitos.
Adotamos nos dois pacientes a laminectomia descompressiva. Além dessa possível forma de tratamento, a laminotomia associada à aspiração endoscópica da gordura e a fenestração interlaminar com descompressão pelo recesso lateral são citadas na literatura. A escolha da técnica depende do nível, da localização e do tamanho da lesão, assim como da experiência do cirurgião. ${ }^{1,4,15-17}$

A redução do peso, da ingesta calórica e a resolução da endocrinopatia de base também são citadas como formas de tratamento.

A maioria dos trabalhos demonstra melhora gradual, acentuada ou completa, do déficit neurológico nos primeiros 2 anos após a cirurgia. ${ }^{15,16,18,19}$ Fato não observado no paciente do terceiro relato, provavelmente pelo tempo transcorrido entre o diagnóstico e o tratamento cirúrgico.

\section{Referências}

1 Robertson SC, Traynelis VC, Follett KA, Menezes AH. Idiopathic spinal epidural lipomatosis. Neurosurgery 1997;41(1):68-74, discussion 74-75

2 Koch CA, Doppman JL, Watson JC, Patronas NJ, Nieman LK. Spinal epidural lipomatosis in a patient with the ectopic corticotropin syndrome. N Engl J Med 1999;341(18):1399-1400

3 Lévy-Weil FE, Feldmann JL. Lipomatose épidurale. Presse Med 2000;29(9):469-475

4 Fassett DR, Schmidt MH. Spinal epidural lipomatosis: a review of its causes and recommendations for treatment. Neurosurg Focus 2004;16(4):E11

5 Dihlmann SW, Mayer HM. [Lumbar epidural lipomatosis]. Z Rheumatol 1995;54(6):417-423

6 López-González A, Resurrección Giner M. Idiopathic spinal epidural lipomatosis: urgent decompression in an atypical case. Eur Spine J 2008;17(Suppl 2):S225-S227

7 Dumont-Fischer D, Rat AC, Saidenberg-Kermanac'h N, Laurent S, Cohen R, Boissier MC. Spinal epidural lipomatosis revealing endogenous Cushing's syndrome. Joint Bone Spine 2002;69(2): $222-225$ 
8 Akhaddar A, Ennouali H, Gazzaz M, Naama O, Elmostarchid B, Boucetta M. Idiopathic spinal epidural lipomatosis without obesity: a case with relapsing and remitting course. Spinal Cord 2008;46(3):243-244

9 Lee SB, Park HK, Chang JC, Jin SY. Idiopathic thoracic epidural lipomatosis with chest pain. J Korean Neurosurg Soc 2011;50(2): 130-133

10 Borré DG. Epidural lipomatosis. J Neurosurg Spine 2007;7(4): 463-464

11 Oikonomou A, Birbilis T, Gymnopoulou E, Prassopoulos P. Paget disease of the spine manifested by thoracic and lumbar epidural lipomatosis: magnetic resonance imaging findings. Spine 2007; 32(25):E789-E792

12 Kuhn MJ, Youssef HT, Swan TL, Swenson LC. Lumbar epidural lipomatosis: the "Y" sign of thecal sac compression. Comput Med Imaging Graph 1994;18(5):367-372

13 Badami JP, Hinck VC. Symptomatic deposition of epidural fat in a morbidly obese woman. AJNR Am J Neuroradiol 1982;3(6): 664-665
14 Haddad SF, Hitchon PW, Godersky JC. Idiopathic and glucocorticoid-induced spinal epidural lipomatosis. J Neurosurg 1991; 74(1):38-42

15 Sairyo K, Sakai T, Higashino K, Hirao B, Katoh S, Yasui N. Minimally invasive excision of lumbar epidural lipomatosis using a spinal endoscope. Minim Invasive Neurosurg 2008; 51(1):43-46

16 Frank E. Endoscopic suction decompression of idiopathic epidural lipomatosis. Surg Neurol 1998;50(4):333-335, discussion 335

17 Payer M, Van Schaeybroeck P, Reverdin A, May D. Idiopathic symptomatic epidural lipomatosis of the lumbar spine. Acta Neurochir (Wien) 2003;145(4):315-320, discussion 321

18 Lisai P, Doria C, Crissantu L, Meloni GB, Conti M, Achene A. Cauda equina syndrome secondary to idiopathic spinal epidural lipomatosis. Spine 2001;26(3):307-309

19 Min WK, Oh CW, Jeon IH, Kim SY, Park BC. Decompression of idiopathic symptomatic epidural lipomatosis of the lumbar spine. Joint Bone Spine 2007;74(5):488-490 